\title{
ATMOSPHERICALLY COMPENSATED SHAPE FROM SHADING ON THE MARTIAN SURFACE: TOWARDS THE PERFECT DIGITAL TERRAIN MODEL OF MARS
}

\author{
M. Hess ${ }^{1, *}$, K. Wohlfarth ${ }^{1}$, A. Grumpe ${ }^{1}$, C. Wöhler ${ }^{1}$, O. Ruesch $^{2}$, B. Wu $^{3}$ \\ ${ }^{1}$ Image Analysis Group, TU Dortmund University, Dortmund, Germany \\ (marcel.hess, kay.wohlfarth, arne.grumpe, christian.woehler)@tu-dortmund.de \\ ${ }^{2}$ ESA/ESTEC, European Space Research and Technology Center, Noordwijk, The Netherlands, ottaviano.ruesch@esa.int \\ ${ }^{3}$ Department of Land Surveying and Geo Informatics, The Hong Kong Polytechnic University, \\ Hung Hom, Kowloon, Hong Kong, bo.wu@ polyu.edu.hk
}

KEY WORDS: Shape from Shading, Stereo, Mars, Atmosphere, Hemispherical directional reflectance

\begin{abstract}
:
We have expanded our existing Shape and Albedo from Shading framework which has primarily been used to generate Digital Terrain Models (DTMs) of the Lunar Surface. The extension consists of an atmospheric model such that the approach can be applied to Mars which is covered by a thin atmosphere. The atmospheric model includes attenuation by the atmosphere, diffuse illumination of the surface and scattering from the atmosphere into the direction of the sensor with physically motivated parameters. To estimate the newly introduced atmospheric parameters without additional CRISM measurements, the radiance image and an initializing surface are used. The initial surface is derived from stereo images and serves two purposes. On the one hand, it is the height constraint of the SfS algorithm and on the other hand, it is used for estimating the atmospheric parameters. Relying on this estimation, the aforementioned Shape and Albedo from Shading method is carried out. The results show a considerable improvement compared to DTMs derived with stereo algorithms. The omnipresent stereo artifacts such as pixel locking and mismatches are smoothed out and small details are reconstructed convincingly. The procedure is then compared to the reconstruction without atmospheric compensation. Images in which shadows are present benefit from this method because shadows can now be described by the diffuse illumination of the surface. The reconstruction results indicate the viability of the approach since it can produce convincing DTMs compared to HiRISE ground truth.
\end{abstract}

\section{INTRODUCTION}

Digital Terrain Models (DTMs) are an essential tool for a variety of remote sensing applications. Geomorphologic analysis, hazard assessment for landing site evaluation, orthoimage rendering, spectral unmixing and accurate temperature modeling, among others, all require or benefit substantially from detailed DTMs. Usually, these DTMs are created with photogrammetric methods. Commonly used stereo algorithms in the planetary science community such as the Ames Stereo Pipeline (Beyer et al., 2018) rely on block matching to find corresponding pixels in the two images and calculate the distance by triangulation. While stereo algorithms yield accurate absolute heights, several artifacts occur which effectively lower the resolution. Extensive parts of planetary surfaces are covered by smooth regolith plains with not many features to match. Block matching is prone to producing mismatches in textureless areas and regions with repetitive structure. Failed matches consequently lead to areas with missing pixels and mismatches lead to an erroneous disparity estimate which yields spikes. Another type of common artifact are stair-like structures due to an effect called pixel locking (Gehrig and Franke, 2016). Spikes and stairs impair the slope estimate, which is crucial for landing site analysis and temperature modeling. It is commonly known that these artifacts all together effectively reduce the resolution of the DTM by an order of magnitude (Alexandrov and Beyer, 2018).

Shape from Shading (SfS, also known as 2D-photoclinometry) on the other hand takes a completely different approach. It employs a physical model of the reflectance behavior to map

\footnotetext{
${ }^{*}$ Corresponding author
}

the image intensities to the slope of the surface. The estimated slopes are numerically integrated to obtain the DTM.

Shape from Shading has the advantage that it has no problems in textureless areas and may yield a resolution which is as high as the resolution of the input image. This does not come without cost. A well-defined reflectance model is needed and larger areas have to be well constrained by a low-resolution DTM. In this approach, we combine the advantages of both: Stereo and SfS.

Early work on SfS (Kirk, 1987, Horn, 1990) has laid the mathematical groundwork for intensity-based surface reconstruction techniques and are the basis for most SfS variants since. Accurate reflectance models are needed to model the received light, but early methods usually assumed a constant surface albedo. The problem to retrieve the shape and albedo from a single image is ill-posed, therefore, additional information must be used. Approaches include machine learning on different Laplacian pyramid representations of the image (Barron and Malik, 2011) or estimating shape and albedo separately (Grumpe and Wöhler, 2014, Wu et al., 2018). These methods make use of regularization terms which use apriori information of the surface to constrain the problem. Shape from Shading has been successfully employed to produce accurate DTMs with pixel level resolution on the Moon (Wu et al., 2018).

When adapting the method to the Martian surface, atmospheric influence has to be considered. The first to employ Shape from Shading on the Martian surface were (Hartt and Carlotto, 1989), for the case of multiple Viking Orbiter images. That approach assumed a constant albedo for a Lambertian surface reflectance 
model. Even though atmospheric effects were mentioned, their method fully neglected atmospheric influence. There are tools that can be used to estimate the influence of the atmosphere on the measured intensity values (Stamnes et al., 1988, Ceamanos et al., 2013). Based on the estimations of the atmosphere derived from CRISM measurements a method has been proposed and successfully applied to CTX imagery (Jiang et al., 2017, Douté et al., 2019). This approach relies on external measurements that are not always available and the albedo of the surface is not estimated on a pixel level, but assumed to be constant over large regions of the image.

Here we expand our previous approach (Wohlfarth et al., 2018) of atmospheric compensation to Shape from Shading based on a physically motivated atmospheric model and the Hapke reflectance model.

\section{METHOD}

In general, the method can be split into three tasks.

1. Construction of an initial DTM and image calibration.

2. Estimation of atmospheric parameters.

3. Shape and Albedo from Shading (SAfS).

The first two steps are executed once before the iterative Shape and Albedo from Shading scheme.

\subsection{Initial DTM creation}

Available data sets like the global HRSC MOLA blend $(200 \mathrm{~m} / \mathrm{pixel})$ have comparably low resolution when compared to CTX (about $6 \mathrm{~m} / \mathrm{pixel}$ ) and especially HiRISE (up to $0.25 \mathrm{~m} / \mathrm{pixel}$ ) images. Therefore, to properly estimate the atmospheric parameters and constrain the SfS procedure, it is necessary to use photogrammetric methods to create an initial DTM. Stereo algorithms produce accurate heights and are consequently a good complementary method to SfS which is especially useful for calculating slopes. Here, the Ames Stereo Pipeline (Beyer et al., 2018) is used to create initial DTMs. It can be easily integrated into the workflow of calibrating the images to I/F values with the ISIS3 software (Gaddis et al., 1997).

\subsection{Reflectance Model}

Shape from Shading aims at finding the orientation of the surface that best describes the measured image intensities. Figure 1 shows the general geometry of a camera observing the surface element illuminated by a light source from a known direction. For the Hapke model, the cosine values of the angles $\vartheta_{e}$ and $\vartheta_{i}$ are used with the following abbreviations:

$$
\mu=\cos \left(\vartheta_{e}\right) \quad \text { and } \quad \mu_{0}=\cos \left(\vartheta_{i}\right)
$$

Hapke's modified isotropic multiple scattering approximation (MIMSA) is a well-established model of the reflectance behavior of particulate planetary surfaces covered by regolith (Hapke, 2002) and will be used for the reflectance modeling.

$r_{\mathrm{d}}\left(\mu, \mu_{0}, g, \omega\right)=\frac{\omega}{4 \pi} \frac{\mu_{0}}{\mu_{0}+\mu}\left[p(g) B_{\mathrm{SH}}(g)+M\left(\mu_{0}, \mu\right)\right] B_{\mathrm{CB}}(g)$.

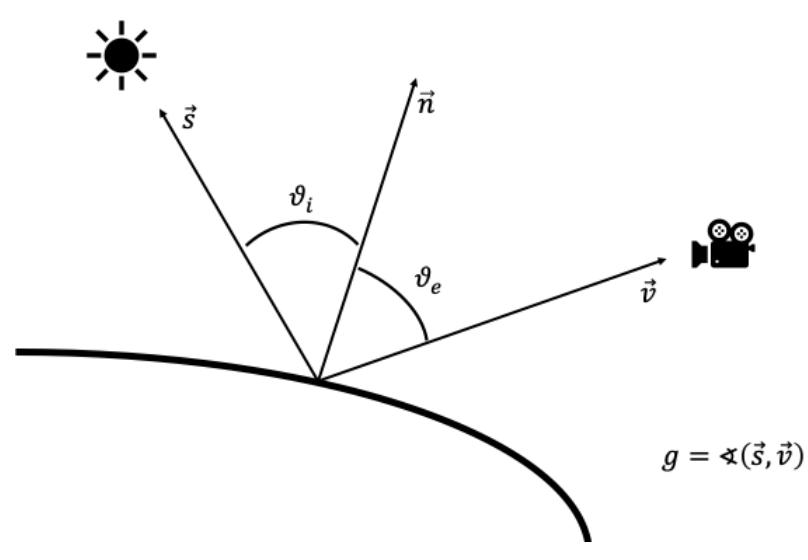

Figure 1. Illumination and viewing geometry.

The single-scattering albedo $\omega$ is estimated in the Shape and Albedo from Shading scheme for each pixel separately. The other parameters of the Hapke model, like the parameters of the phase function and the Shadow Hiding and Coherent Backscatter correction factors are set to default values (Warell, 2004).

\subsection{Model of the Atmosphere}

In general, the Top of Atmosphere (TOA) radiance measured at the sensor consists of several contributing factors. In a simplifying manner, we choose the three most important contributors which are (1) light that is directly reflected from the surface into the direction of the sensor, (2) light that is reflected from the atmosphere down to the surface diffusely illuminating it and then again reflected into the direction of the spacecraft and (3) light that is reflected by the atmosphere into the direction of the sensor (Schowengerdt, 2007). Electromagnetic waves traveling through a medium are attenuated depending on the optical depth $\tau$ of that medium and the air mass it has to pass. This effect can be modeled with the Lambert-Beer law:

$$
E_{\text {ground }}=E_{\text {sun }} \mathrm{e}^{-\frac{\tau_{1}}{\mu_{0 s}}}
$$

The solar irradiance $E_{\text {sun }}$ is attenuated on the way to the surface depending on the optical depth $\tau$ and the air-mass defined by the cosine of the solar incidence zenith angle relative to a flat surface $\mu_{0 s}$. The TOA radiance contribution of light that diffusely illuminates the surface can be calculated by integrating the bidirectional reflectance function $r_{\mathrm{d}}$ over the upper hemisphere:

$$
r_{\mathrm{hd}}(\mu)=\int_{\mu_{0}=0}^{1} \int_{\varphi=0}^{2 \pi} r_{d}\left(\mu_{0}, \mu, g\right) d \varphi d \mu_{0}
$$

The angle $\varphi$ denotes the azimuth angle, and the phase angle $g$ depends on $m u$ and $m u_{0}$. The total radiance measured at the sensor then becomes:

$$
I_{\mathrm{TOA}}=\left(\mathrm{e}^{-\frac{\tau}{\mu_{0 s}}} r_{\mathrm{d}}\left(\vartheta_{i}, \vartheta_{e}, g\right)+\zeta r_{\mathrm{hd}}\left(\vartheta_{e}\right)\right) \mathrm{e}^{-\frac{\tau}{\mu_{s}}}+\chi
$$

where

$$
\begin{aligned}
& \tau=\text { optical depth } \\
& \zeta=\text { diffuse illumination } \\
& \chi=\text { path scattered radiance } \\
& r_{\mathrm{d}}=\text { bidirectional reflectance function } \\
& r_{\mathrm{hd}}=\text { hemispherical directional reflectance function }
\end{aligned}
$$

The parameters for the atmosphere are estimated once before the Shape and Albedo from Shading based on the initial DTM 
and the image intensities. Shadows can be used to estimate the parameters $\zeta$, and $\chi$ because all light received by the sensor is only due to diffuse illumination of the surface and radiance scattered by the atmosphere directly. We obtain the optical depth $\tau$ by fitting such that the combined reflectance and atmospheric model directly maps the slopes, which are derived from the initial stereo DTM to the radiance image. To account for the stereo artifacts the fit is performed at a slightly lower resolution on a large number of data points. We find that best fits on several different images yield values for the optical depth between 0.35 and 0.5 which is consistent with other studies (Petrova et al., 2012).

\subsection{Shape from Shading}

Shape from Shading is discussed in many publications. In this work, we have used our existing Shape from Shading framework (Grumpe and Wöhler, 2014). This method has been successfully applied to the lunar surface, primarily to $M^{3}$ (Grumpe and Wöhler, 2014, Grumpe et al., 2014) and LROC NAC (Kim et al., 2016, Wu et al., 2018) imagery. Minimizing the intensity error of the measured radiance and the modeled radiance with the inclusion of the atmospheric model requires a few regularization parameters to stabilize the procedure and solve the underdetermined problem. First, the integrability error according to (Horn, 1990) is used to ensure a continuous surface. Secondly, the initializing DTM is used as a constraint by comparing the absolute heights (Shao et al., 1991, Grumpe and Wöhler, 2014) and the gradient values (Grumpe et al., 2014) to the current estimates. These comparisons to the initializing surface are carried out in a lower frequency domain to be scale consistent.

\section{RESULTS}

Because HiRISE images have the highest resolution available, there is no DTM data that can be used as ground truth for validation. Therefore, we show the results for a small region of a CTX image that has HiRISE coverage available as well and use the stereo-derived HiRISE DTM as ground truth.

\subsection{Crater near Alba Patera}

The region of interest is taken from a crater at the north-east of Alba Patera (Jiang et al., 2017). The image pair from Table 1 was used to construct the stereo DTM in the Ames Pipeline and the image G20_025970_2217_XN_41N102W was employed for the Shape from Shading algorithm. Figure 2 shows the image of the region of interest and the yellow line indicates the profile in the center of the image with a variety of structures that will be investigated further. Figure 3 shows the color-coded DTM of the region which is overlain with a shading of the surface for constant albedo and illuminated from the west at $60^{\circ}$ incidence of the sun. While the general structure and heights are well reproduced, the stereo artifacts are clearly visible. This DTM was then again filtered and the resolution was reduced to remove the artifacts and was then used as an initializing surface for the SfS procedure.

Figure 4 shows the DTM constructed by the new method with atmospheric compensation. It is shaded with a constant albedo. The small structures apparent in the image are clearly visible.

To investigate the results further, it is necessary to have a reference that can be used for validating the results. For this purpose, the HiRISE stereo DTMs can be used after the DTM was

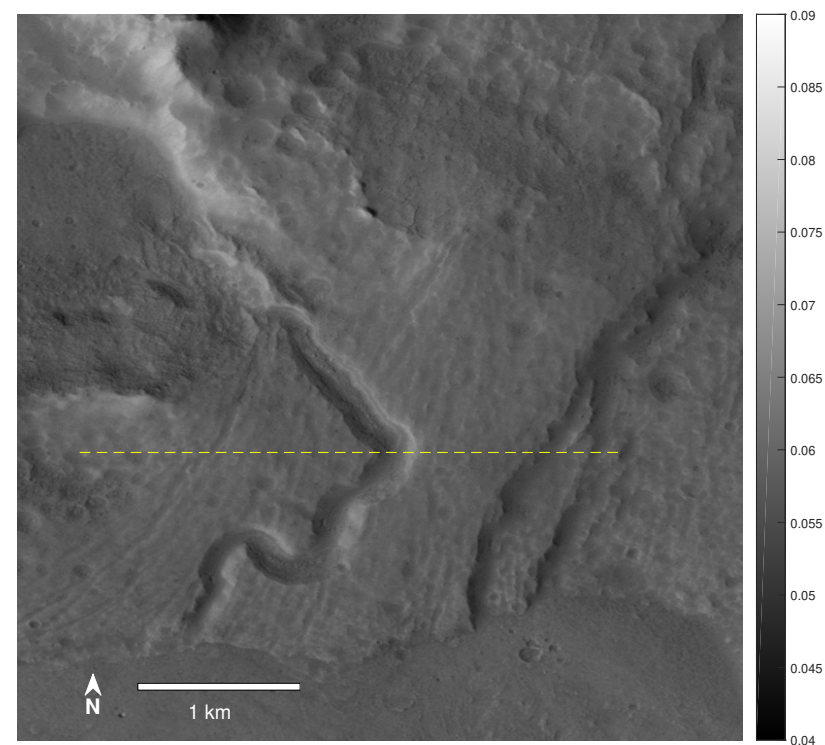

Figure 2. Image in $I / F$ of the region of interest taken from CTX image G20_025970_2217_XN_41N102W. The yellow dashed line indicates the profile which will be investigated further in the following figures.

downsampled to CTX resolution and filtered to remove severe artifacts. We used the HiRISE stereo DTM as ground truth. Figure 7 shows the profile comparison of the yellow dashed line in Figure 2. As an additional comparison, the results are compared to the DTM created without atmospheric compensation. It can be seen, that the Shape from Shading method significantly improves the initial DTM and a lot of the smaller structures like the hills on the left and the depth of the rift are well reconstructed. The slope of the profile is plotted in Figure 8. The RMSE values are shown in Table 2 and also show that the SfS methods improve over the initial DTM and the atmospherically compensated DTM performs slightly better than the method without atmospheric compensations.

Another illustration that shows the difference between stereo and Shape from Shading DTMs is to plot the slope maps of the scene. The slope is the arccosine of the z-component of the surface normal vector of length 1 . In Figure 5 the slope map of the stereo DTM is displayed, which shows that there are some artifacts clearly visible in the bottom left and on the edges of the rim in the center. The stair-like structures are also clearly visible, which are due to pixel locking which leads to exaggerated slope estimates. These artifacts and stair-like structures disappear in the Shape from Shading DTM and the slopes become more realistic.

\section{CONCLUSION}

We have included atmospheric modeling based on the Hapke hemispherical directional reflectance and the Lambert-Beer law

\begin{tabular}{l|l|l|l} 
Image & Resolution & Latitude & Longitude \\
\hline $\begin{array}{l}\text { G20_025904_2209 } \\
\text { _N_40N102W }\end{array}$ & $5.98 \mathrm{~m} / \mathrm{px}$ & $42.857^{\circ} \mathrm{N}$ & $-102.819^{\circ} \mathrm{E}$ \\
\cline { 1 - 1 } $\begin{array}{l}\text { G20_025970_2217 } \\
\text { _XN_41N102W }\end{array}$ & $5.98 \mathrm{~m} / \mathrm{px}$ & $42.932^{\circ} \mathrm{N}$ & $-102.744^{\circ} \mathrm{E}$
\end{tabular}

Table 1. General information about the images. 


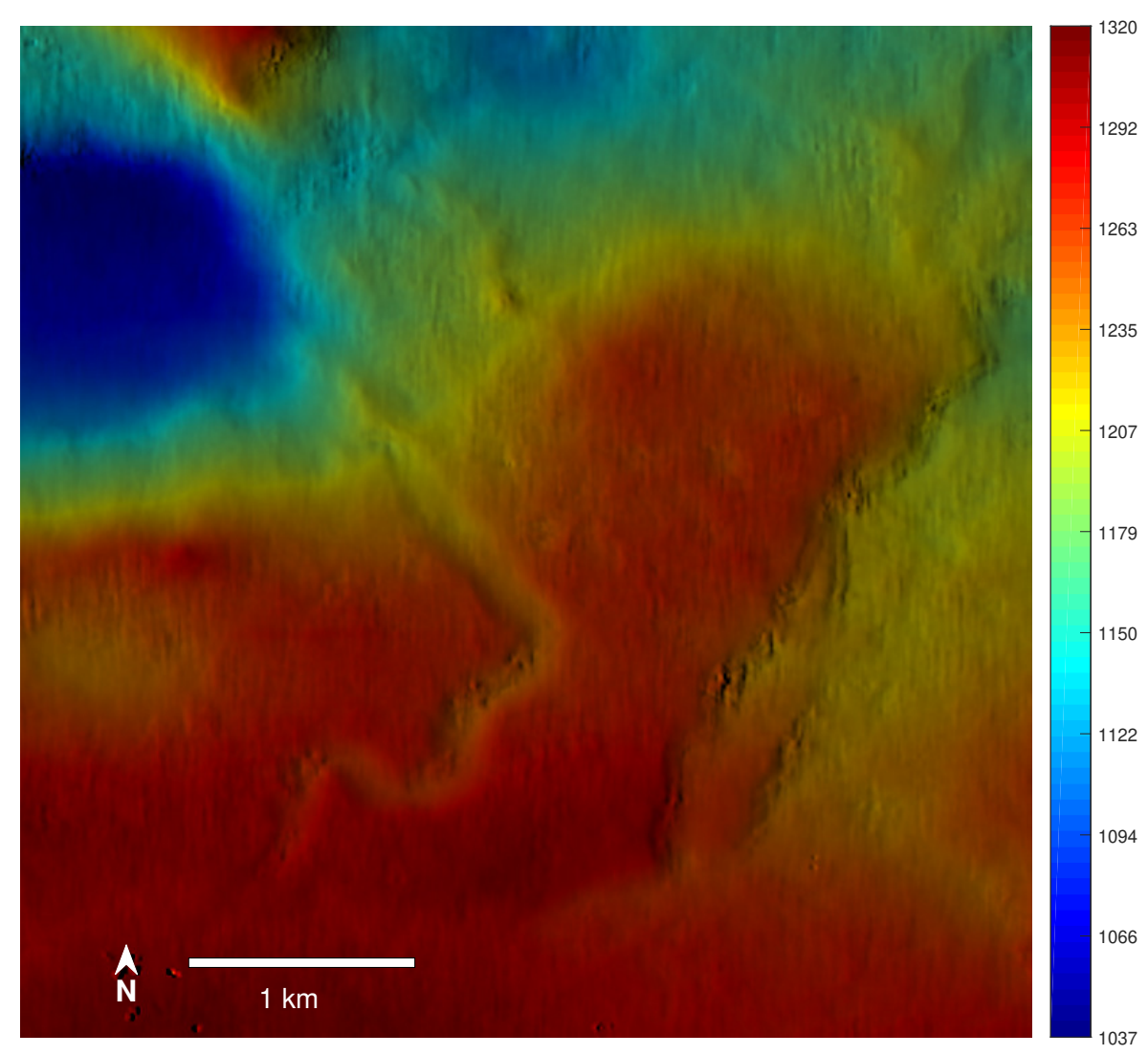

Figure 3. Color-coded stereo DTM created with the Ames Stereo Pipeline. Note that the DTM is already sub sampled by a factor of two by default.

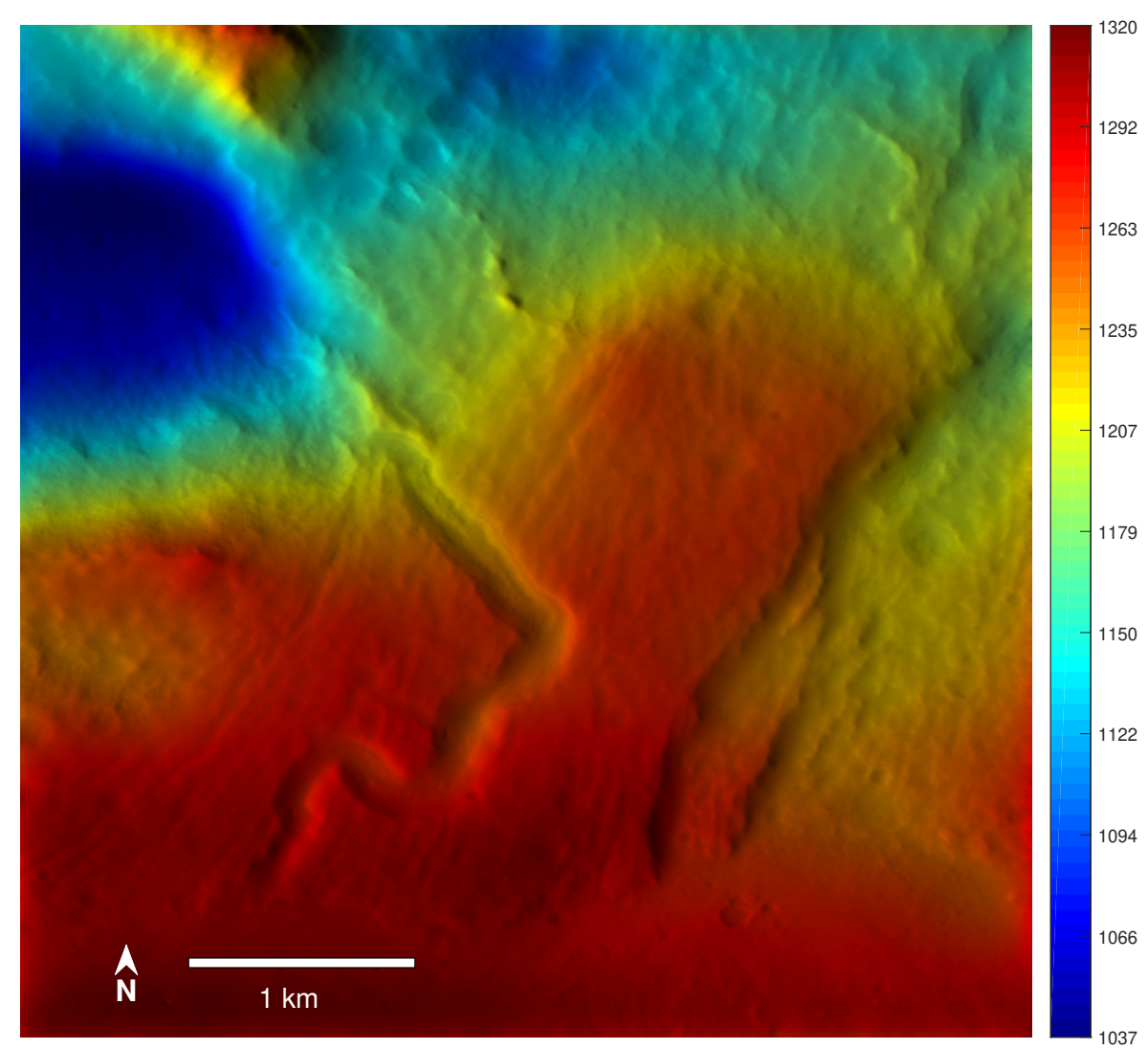

Figure 4. Color-coded DTM produced with the new method including atmospheric compensation. 


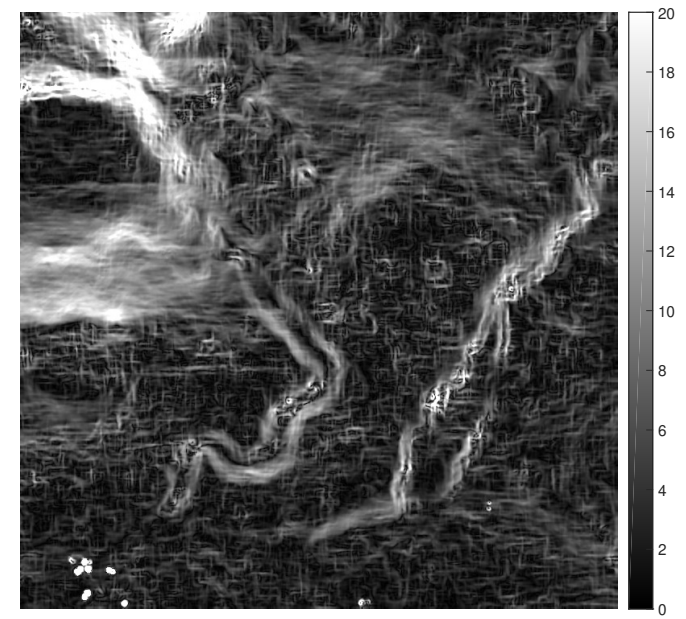

Figure 5. Slope map of the stereo DTM.

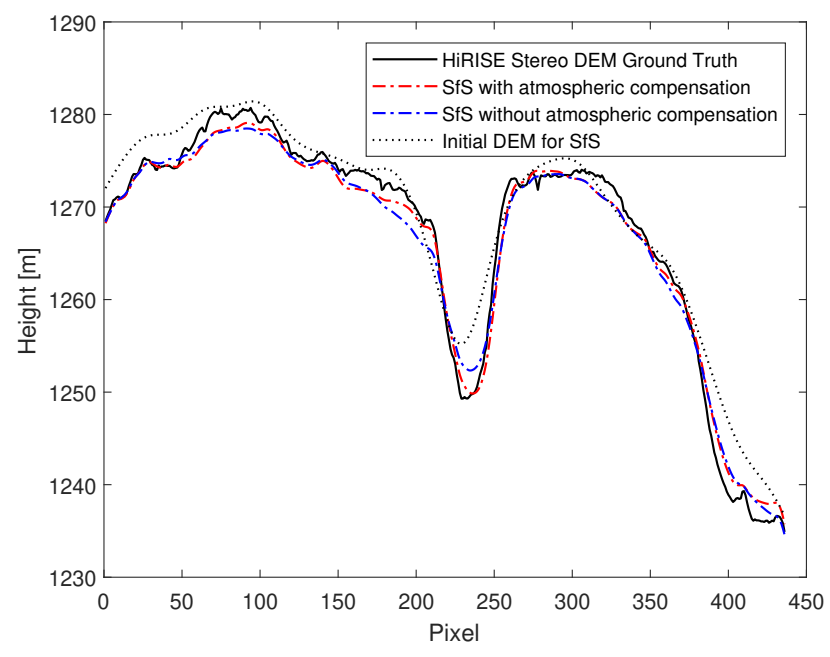

Figure 7. Height profile produced with the different methods.

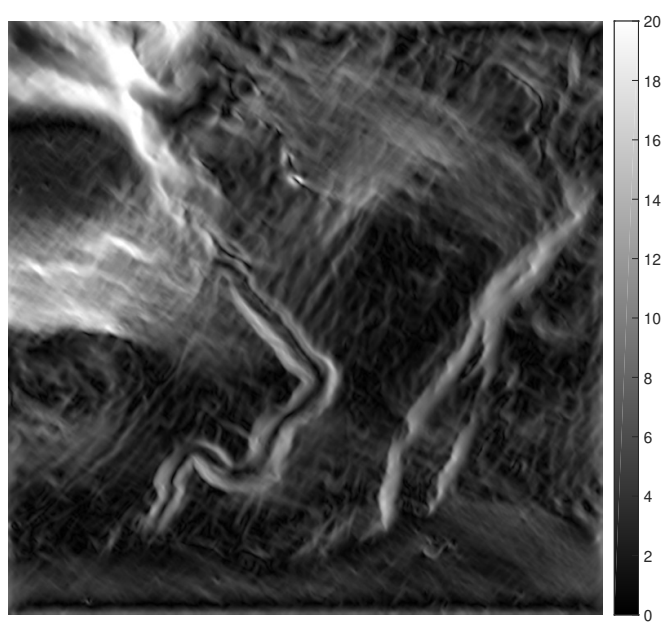

Figure 6. Slope map of the SAfS DTM.

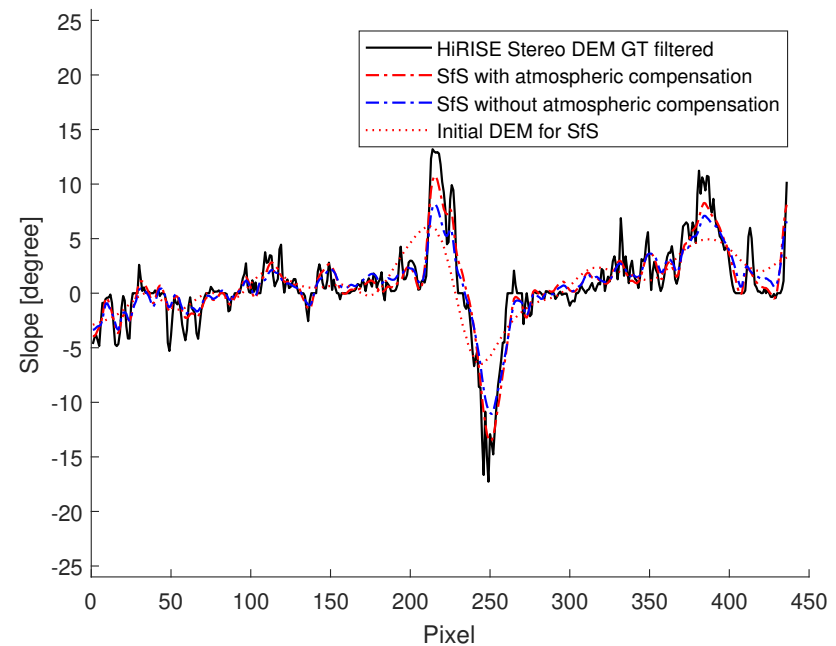

Figure 8. Slope of the profile from Figure 7. 


\begin{tabular}{l|l|l} 
DTM & RMSE height & RMSE slope \\
\hline Initial DTM & $2.07 \mathrm{~m}$ & $6.52^{\circ}$ \\
\hline $\begin{array}{l}\text { Without atmospheric } \\
\text { compensation }\end{array}$ & $1.49 \mathrm{~m}$ & $2.80^{\circ}$ \\
\hline $\begin{array}{l}\text { With atmospheric } \\
\text { compensation }\end{array}$ & $1.27 \mathrm{~m}$ & $1.89^{\circ}$ \\
\hline
\end{tabular}

Table 2. Comparison of different DTMs to the HiRISE stereo DTM ground truth.

to adapt our existing Shape and Albedo from Shading framework to the Martian surface. The parameters for the model are physically motivated and the albedo is estimated on pixel level. On a selected CTX region, the results look convincing and the validation with a HiRISE-derived ground truth stereo DTM yield a good correspondence. While stereo algorithms already yield accurate heights in their resolution domain, the slope estimates are significantly better for the Shape from Shading methods. The incorporation of an initial DTM of lower resolution as regularization terms for the Shape and Albedo from Shading algorithm ensures that this additional information is used and the general structure of the surface is maintained, while not penalizing the reconstruction of small structures. All in all, the construction of DTMs by a combination of Shape and Albedo from Shading with a correction for atmospheric effects yields encouraging results for the Martian surface.

\section{REFERENCES}

Alexandrov, O., Beyer, R. A., 2018. Multiview Shape-FromShading for Planetary Images. Earth and Space Science, 5, 652666.

Barron, J. T., Malik, J., 2011. High-Frequency Shape and Albedo from Shading using Natural Image Statistics. Computer Vision and Pattern Recognition (CVPR), 2521 - 2528.

Beyer, R. A., Alexandrov, O., McMichael, S., 2018. The Ames Stereo Pipeline: NASA's Open Source Software for Deriving and Processing Terrain Data. Earth and Space Science.

Ceamanos, X., Douté, S., Fernando, J., Schmidt, F., Pinet, P., Lyapustin, A., 2013. Surface reflectance of Mars observed by CRISM/MRO: 1. Multi-angle approach for retrieval of surface reflectance from CRISM observations (MARS-ReCO). 118, 514-533.

Douté, S., Conway, S., Massé, M., 2019. Small Scale Topographical Characterizationof Jezero Crater Region,Mars. 50th Lunar and Planetary Science Conference, Lunar and Planetary Institute, Houston, Abstract \#2528.

Gaddis, L., Anderson, J., Becker, K., Becker, T., Cook, D., Edwards, K., Eliason, E., Hare, T., Kieffer, H., Lee, E. M., Mathews, J., Soderblom, L., Sucharski, T., Torson, J., McEwen, A., Robinson, M., 1997. An Overview of the Integrated Software for Imaging Spectrometers (ISIS). Lunar and Planetary Science Conference, Lunar and Planetary Inst. Technical Report, $28,387$.

Gehrig, S., Franke, U., 2016. Stereovision for ADAS. Handbook of Driver Assistance Systems, Springer International Publishing, chapter 21, 495-524.
Grumpe, A., Belkhir, F., Wöhler, C., 2014. Construction of lunar DEMs based on reflectance modelling. Advances in Space Research, 53, 1735-1767.

Grumpe, A., Wöhler, C., 2014. Recovery of elevation from estimated gradient fields constrained by digital elevation maps of lower lateral resolution. ISPRS Journal of Photogrammetry and Remote Sensing, 94, 37 - 54.

Hapke, B., 2002. Bidirectional reflectance spectroscopy: 5. The Coherent Backscatter Opposition Effect and Anisotropic Scattering. Icarus, 157, 523-534.

Hartt, K., Carlotto, M., 1989. A method for shape-from-shading using multiple images acquired under different viewing and lighting conditions. Proceedings CVPR '89: IEEE Computer Society Conference on Computer Vision and Pattern Recognition, 53-60.

Horn, B. K. P., 1990. Height and Gradient from Shading. International Journal of Computer Vision, 5, 37-75.

Jiang, C., Douté, S., Luo, B., Zhang, L., 2017. Fusion of photogrammetric and photoclinometric information for highresolution DEMs from Mars in-orbit imagery. ISPRS Journal of Photogrammetry and Remote Sensing, 130, 418-430.

Kim, K., Wöhler, C., Hyeok Ju, G., Lee, S. R., P. Rodriguez, A., Berezhnoy, A., Gasselt, S., Grumpe, A., Aymaz, R., 2016. Korean Lunar Lander - Concept Study for Landing-Site Selection for Lunar Resource Exploration. ISPRS - International Archives of the Photogrammetry, Remote Sensing and Spatial Information Sciences, XLI-B4, 417-423.

Kirk, R. L., 1987. III. A fast finite-element algorithm for twodimensional photoclinometry. $\mathrm{PhD}$ thesis, California Institute of Technology.

Petrova, E.V., Hoekzema, N.M., Markiewicz, W.J., Thomas, N., Stenzel, O.J., 2012. Optical depth of the Martian atmosphere and surface albedo from high-resolution orbiter images. Planetary and Space Science, 60, 287 - 296. Titan Through Time: A Workshop on Titanas Formation, Evolution and Fate.

Schowengerdt, R. A., 2007. Remote Sensing - Models and Methods for Image Processing. Waltham, MA: Elsevier Science.

Shao, Min, Chellappa, Rama, Simchony, Tal, 1991. Reconstructing a 3-D depth map from one or more images. CVGIP: Image Understanding, 53, 219 - 226.

Stamnes, K., Tsay, S., Wiscombe, W., Jayaweera, K., 1988. Numerically stable algorithm for discrete-ordinate-method radiative transfer in multiple scattering and emitting layered media. Appl. Opt., 27, 2502-2509.

Warell, J, 2004. Properties of the Hermean regolith: IV. Photometric parameters of Mercury and the Moon contrasted with Hapke modelling. Icarus, 167, 271 - 286.

Wohlfarth, K. S., Liu, W. C., Wu, B., Grumpe, A., Wöhler, C., 2018. High resolution digital terrain models of the martian surface: Compensation of the atmosphere on ctx imagery. 49th Lunar and Planetary Science Conference, Lunar and Planetary Institute, Houston, Abstract \#2498. 
Wu, B., Liu, W. C., Grumpe, A., Wöhler, C., 2018. Construction of pixel-level resolution DEMs from monocular images by shape and albedo from shading constrained with low-resolution DEM. ISPRS Journal of Photogrammetry and Remote Sensing, 140. 